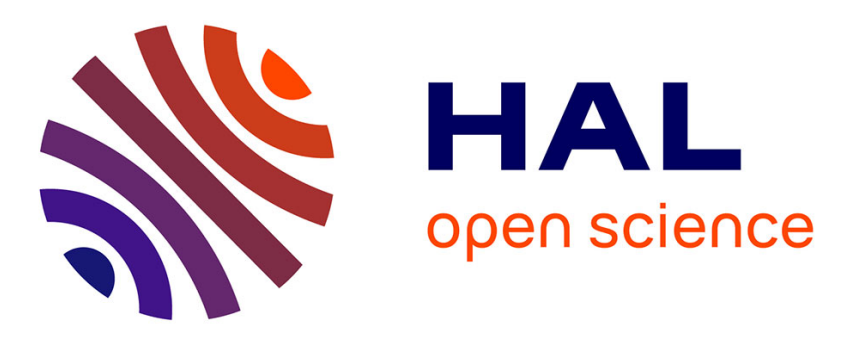

\title{
A modified servo-hydraulic machine for testing at intermediate strain-rates
}

Ramzi Othman, Pierrick Gu'Egan, Georges Challita, Franck Pasco, Daniel Lebreton

\section{- To cite this version:}

Ramzi Othman, Pierrick Gu'Egan, Georges Challita, Franck Pasco, Daniel Lebreton. A modified servo-hydraulic machine for testing at intermediate strain-rates. International Journal of Impact Engineering, 2008, 36 (3), pp.460-467. 10.1016/j.ijimpeng.2008.06.003 . hal-00550175

\section{HAL Id: hal-00550175 https://hal.science/hal-00550175}

Submitted on 24 Dec 2010

HAL is a multi-disciplinary open access archive for the deposit and dissemination of scientific research documents, whether they are published or not. The documents may come from teaching and research institutions in France or abroad, or from public or private research centers.
L'archive ouverte pluridisciplinaire HAL, est destinée au dépôt et à la diffusion de documents scientifiques de niveau recherche, publiés ou non, émanant des établissements d'enseignement et de recherche français ou étrangers, des laboratoires publics ou privés. 


\section{Accepted Manuscript}

Title: A modified servo-hydraulic machine for testing at intermediate strain-rates

Authors: Ramzi Othman, Pierrick Gu'egan, Georges Challita, Franck Pasco, Daniel LeBreton



PII: $\quad$ S0734-743X(08)00122-X

DOI: $\quad$ 10.1016/j.jijmpeng.2008.06.003

Reference: IE 1604

To appear in: International Journal of Impact Engineering

Received Date: 28 June 2007

Revised Date: 9 June 2008

Accepted Date: 10 June 2008

Please cite this article as: Othman R, Gu'egan P, Challita G, Pasco F, LeBreton D. A modified servo-hydraulic machine for testing at intermediate strain-rates, International Journal of Impact Engineering (2008), doi: 10.1016/j.jijmpeng.2008.06.003

This is a PDF file of an unedited manuscript that has been accepted for publication. As a service to our customers we are providing this early version of the manuscript. The manuscript will undergo copyediting, typesetting, and review of the resulting proof before it is published in its final form. Please note that during the production process errors may be discovered which could affect the content, and all legal disclaimers that apply to the journal pertain. 


\title{
A modified servo-hydraulic machine for testing at intermediate strain-rates
}

\author{
Ramzi Othman ${ }^{\mathrm{a}}$, Pierrick Guégan ${ }^{\mathrm{a}}$, Georges Challita ${ }^{\mathrm{a}, \mathrm{b}}$, \\ Franck Pasco $^{\mathrm{a}}$, Daniel LeBreton ${ }^{\mathrm{a}}$ \\ ${ }^{\mathrm{a}}$ Institut de Recherche en Génie Civil et Mécanique, Ecole Centrale de Nantes, 1 \\ Rue de la Noё BP 92101, 44321 Nantes cedex 3, France \\ ${ }^{\mathrm{b}}$ Département de Mécanique, Faculté de Génie Branche 2, Université Libanaise, \\ Beyrouth Liban.
}

\begin{abstract}
Assessing the mechanical properties of materials at intermediate strain rates (1$200 / \mathrm{s}$ ) is one of the major concerns in impact engineering. Servo-hydraulic machines are usually used at this range of strain rates. However, detrimental oscillations appear on the measured load for strain rates above 10/s. In this paper, we present a modified servo-hydraulic machine. The load is measured by a Hopkinson bar-like technique. Besides, the BCGO wave separation method is used to overcome the limited test duration problem. Moreover, the strain in the sample is measured using Digital Speckle Photographs (DSP) which are captured via high-speed video camera. This modified machine is an equivalent and alternative solution to the slow bar technique. It is applied to aluminium $2017 \mathrm{~T} 4$ alloy at strain-rates of approximately 100/s. Compared to the piezoelectric force sensor, the Hopkinson bar-like method gives less-oscillating signals. The sample attaching device is also taken into account. It is considered as a rigid mass. This simple assumption is valid up to strain-rates
\end{abstract}


of some hundreds per second.

Key words: Intermediate strain rate, wave separation, Servo-hydraulic machine, Hopkinson bar, Aluminium.

PACS:

\section{Introduction}

In several engineering applications, such as crashworthiness, anti-seismic protection [1] and dynamic forming processes [2], materials may be subject to intermediate strain rates (1-200/s). The choice of material constitutive equations and the identification of the model parameters depend on the measurement of mechanical properties at these strain rates. However, no reliable technique exists at this range of strain rates.

Conventional quasi-static machines are valid up to strain rates of $0.1 / \mathrm{s}$. Split Hopkinson bar (SHB) method is applied to tests at strain rates ranging from 200 to 5000/s. Servo-hydraulic machines have been regarded as a solution to characterisation of materials at intermediate strain rates. Nevertheless, measurements are distorted by the inertia and elastic response of the experimental set-up. The measured load exhibits some oscillations which are due to the natural frequencies of the experimental structure [2]. Consequently, servo-hydraulic machines give reliable measurements only at strain rates lower than 10/s [3].

Alternatively to servo-hydraulic machines, dropweight towers can be used to generate data at intermediate strain-rates [4-9]. However, the load is measured

Email address: ramzi.othman@ec-nantes.fr (Ramzi Othman). 
with a piezoelectric force sensor [3] as in servo-hydraulic machines. Unless special care is paid to the design of the load cell or to the processing of load cell signals, large oscillations appear $[1,10]$. These oscillations may be large enough to obscure the real force applied to the specimen [3]. Therefore, dropweight towers are generally applied to the measurement of the crash response of structures $[10,11]$.

The elastic response of the experimental set-up is one of the major problems in dynamic tests. The main asset of the SHB technique is the simplicity of the experimental set-up: two (visco-)elastic bars. Therefore, the (visco-)elastic response is simply and finely taken into account. The SHB is limited to high strain rates, on account of the limited test duration. Assuming that the sample is deformed at a constant strain rate $\dot{\epsilon}$, the maximum recorded strain is therefore $\epsilon_{\max }=\dot{\epsilon} \Delta t$, where $\Delta t$ is the test duration. For conventional SHB the test duration is rarely above $500 \mu \mathrm{s}$. Hence, at a mean strain rate of $100 / \mathrm{s}$, the maximum measured strain is $5 \%$. This leads many authors [12-25] to develop wave separation techniques which increase the test duration.

A second problem is also encountered in intermediate strain rate tests with SHB. Indeed, low impact velocities of the striker are needed. This yields low kinetic energy in the striker which is not sufficient to induce large strains in the sample. Thus, an alternative loading technique is required. A hydraulic jack is proposed in [15,26-28]. It applies a continuous load during the test duration. Alternatively, LeBlanc \& Lassila [3] proposed a hybrid technique which combines the loading capabilities of a servo-hydraulic machine and the measurement technique of a Hopkinson bar. The assets of this method are: (i) continuous loading during the test which supplies the sufficient energy to induce important strain in the sample at intermediate strain rates and (ii) 
a reliable force and consequently stress measurement. However, in this work, only one gauge station is used on the bar (regarded as an output bar in a SHB technique). The gauge station is not far from the sample-bar interface. Therefore, the test duration is approximately twice the duration of a conventional SHB, i.e., no more than $1 \mathrm{~ms}$, that is not enough yet.

To maximize the test duration, Quirion \& Lesaffre [29] proposed the application of a wave separation technique to servo-hydraulic machines. They instrumented the anvil with two gauges stations. They recovered the force using the wave separation technique developed in $[12,13]$. However, two problems were not considered. The first one is that the Lundberg-Henchoz-Yanagihara method $[12,13]$ neglects wave dispersion. The anvil has a diameter of $22 \mathrm{~mm}$. Consequently, geometry effects on wave dispersion are important and can not be neglected. Thus, an alternative wave separation technique, which takes into account wave dispersion, should have been used. The second problem is relative to the strain measurements. The gauge stations are cemented approximately $30 \mathrm{~mm}$ from the anvil ends which was only 1.36 times the diameter. Thus, the assumption of one-dimensional propagation of waves was not valid. Indeed, it is usually recommended to record strain at distances above 10 times the diameter.

In this paper, the hybrid technique of LeBlanc and Lassila [3] is improved by using a wave separation technique. Consequently, the test duration is highly increased. Instead of wave separation techniques one can use an inverse approach as proposed in $[2,30]$. Nevertheless, this approach has some drawbacks: (i) it needs a parametric formulation of the recovered load; (ii) it is time consuming compared to wave separation techniques; (iii) it is based on an optimisation problem which could yield a local minimum and not a global one. 


\section{ARTICLE IN PRESS}

Hence, the obtained solution has not usually a physical sense because of (i) and (iii).

This paper aims at applying a long duration Hopkinson bar method to servohydraulic machines. Therefore, a wave separation method is needed. In the following section, a critical review of wave separation techniques is presented. The objective of this section is to figure out the best method. In the section 3, we describe, how the selected method, can be applied to servo-hydraulic machines. The fourth section, gives results obtained with this new technique on aluminium 2017 T4 alloy. Less-oscillating load measurements are obtained. Furthermore, the test duration restraint is subdued.

\section{Critical review of wave separation techniques}

Let us consider a straight uniform visco-elastic bar. We denote $\tilde{\epsilon}(x, \omega), \tilde{V}(x, \omega)$ and $\tilde{N}(x, \omega)$ the Fourier transforms of the strain $\epsilon(x, t)$, the particle velocity $V(x, t)$ and the force $N(x, t)$, respectively, at any cross-section $x$. Assuming a one-dimensional wave propagation and taking into account the $3 \mathrm{D}$ geometric effects on the wave dispersion, these Fourier transforms are given by:

$$
\begin{aligned}
& \tilde{\epsilon}(x, \omega)=F(\omega) e^{-i \xi(\omega) x}+D(\omega) e^{i \xi(\omega) x} \\
& \tilde{V}(x, \omega)=\frac{\omega}{\xi(\omega)}\left(-F(\omega) e^{-i \xi(\omega) x}+D(\omega) e^{i \xi(\omega) x}\right) \\
& \tilde{N}(x, \omega)=E(\omega) A\left(F(\omega) e^{-i \xi(\omega) x}+D(\omega) e^{i \xi(\omega) x}\right)
\end{aligned}
$$

where $x, A, E(\omega), \xi(\omega), F(\omega)$ and $D(\omega)$ are the position of the considered cross-section, the cross-sectional area of the bar, the complex 
Young's modulus, the wave dispersion relation, the forward wave and the downward wave, respectively.

Eqs. (1-3) mean that the force and the particle velocity can be recovered at any cross-section of the bar if the four following frequencydependent functions are known: $E(\omega), \xi(\omega), F(\omega)$ and $D(\omega)$. Fortunately, the two former functions are characteristics of the bar and have to be determined just once. Furthermore, the two waves $F(\omega)$ and $D(\omega)$ can be recovered from a reduced number of measurements on the bar. Two equations should be sufficient to do so, since we have only two unknown functions: $F(\omega)$ and $D(\omega)$. Therefore, the strain is measured at two different cross-sections, say $x_{1}$ and $x_{2}$. Two equations can be derived from Eq. (1):

$$
\begin{aligned}
& \tilde{\epsilon}_{1}(\omega)=\tilde{\epsilon}\left(x_{1}, \omega\right)=F(\omega) e^{-i \xi(\omega) x_{1}}+D(\omega) e^{i \xi(\omega) x_{1}}, \\
& \tilde{\epsilon}_{2}(\omega)=\tilde{\epsilon}\left(x_{2}, \omega\right)=F(\omega) e^{-i \xi(\omega) x_{2}}+D(\omega) e^{i \xi(\omega) x_{2}},
\end{aligned}
$$

where $\tilde{\epsilon}_{1}(\omega)$ and $\tilde{\epsilon}_{2}(\omega)$ are the Fourier transforms of the measured strain at the two cross-sections. If these two equations are independent, we can simply calculate the two waves, as suggested in [15]. Unfortunately, the determinant of Eqs. (4-5) could be equal to zero (for elastic bars) or nearly be equal to zero (for viscoelastic bars). In the case of elastic bars, the determinant of Eqs. (4-5) equals zero for a set of resonant frequencies defined by

$$
\Omega_{e}=\left\{\omega_{n} ; \xi\left(\omega_{n}\right)\left(x_{2}-x_{1}\right)=n \pi, \forall n \in I N\right\}
$$

In the case of a viscoelastic bar, the set of resonant frequencies is theoretically 
reduced to $\Omega_{v}=\{0\}$. However, the denominator has a low value for all the other frequencies in $\Omega_{e}$.

Using the one-dimensional wave equation for rods, Lundberg \& Henchoz [12] and Yanagihara [13] developed independently a time domain solution. The main limitation of this method is that it does not take into account wave dispersion effects. Therefore, it is limited to slender elastic bars. Zhao and Gary $[14,15]$ proposed a method which takes into account wave dispersion. It uses also two strain measurements. In this method, each measured strain is split into time intervals to operate successive shifting. The length of each interval is equal to twice the time needed by the wave to go from one gauge to the other. This time depends on the wave velocity which is frequency-dependent. Therefore, the interval length should depend on frequency. However, in the Zhao and Gary works $[14,15]$, this time is inevitably taken frequency independent. As the method is iterative, errors will cumulate. Consequently, its validity is limited to approximately some hundreds of iterations. The test duration is considerably increased (it can reach $200 \mathrm{~ms}$ ) but still limited.

The solutions presented in [12-15] did not thoroughly take into account wave dispersion. Hence, Bacon [16] suggested the use of a hybrid method to subdue this problem. The wave separation was performed in the frequency and time domain. Since the problem with Eqs. (4-5) concerns only the frequencies that are in $\Omega_{e}$, Bacon applied these two equations to the frequencies $\omega \notin \Omega_{e}$. For the resonant frequencies $\left(\omega_{n} \in \Omega_{e}\right)$, he used a time solution which is equivalent to the solution presented in $[12,13]$. Thus, this method overcomes the problem with resonant frequencies and includes wave dispersion effects. In line with 
this work, Zhao \& Lok [21] deal separately with the resonant frequencies. On one hand, they used also Eqs. (4-5) outside $\Omega_{e}$. On the other hand, the frequencies $\omega_{n} \in \Omega_{e}, F\left(\omega_{n}\right)$ and $D\left(\omega_{n}\right)$ are undefined fractions of type $0 / 0$. Hence, $F\left(\omega_{n}\right)$ and $D\left(\omega_{n}\right)$ are calculated using the Hopital's rule. The method presented in [16] recovers the two elementary waves in the resonant frequencies by an iterative method. Therefore, it is time consuming. On the contrary, the method proposed in [21] is a direct frequency method. Nevertheless, it uses numerical differentiation of measured signals. This amplifies errors mainly at high frequencies. The maximum test duration presented in [16] is $6 \mathrm{~ms}$ and in [21] is 2ms. To solve the problem of dependency in Eqs. (4-5), Bussac et al. $[19,20]$ proposed the integration of Fourier transforms in the lower half-plane of the frequency complex space $(\Im(\omega) \prec 0)$. This numerical tool is possible because the Fourier transforms of the measured strains $\tilde{\epsilon}_{1}(\omega)$ and $\tilde{\epsilon}_{2}(\omega)$ are analytical bounded functions in the lower frequency half-plane. Moreover, the wave dispersion relation is assumed to be an analytical function in the same half-plane and maps this half-plane to itself.

The wave separation methods presented in $[16,22]$ and the first method presented in [20] use only two strain measurements. This would be enough if the measured strains were perfectly free from noise, which is impossible in actual experimental situations. Besides, these methods, as deconvolution techniques, are an ill-posed problem. Consequently, initial noise is amplified when rebuilding the force and the particle velocity [18]. It was concluded in [18] that initial noise is highly amplified by the recovering process in the neighbourhood of the resonant frequencies. It was then suggested in $[19,20,24]$ the use of redundant measurements. Jacquelin \& Hamelin [24] presented a 3-strain method. It assumes a particular relation 
between the strain gauge positions. Bussac et al. $[19,20]$ proposed the analysis of the measured strains, and eventually velocities, by the maximum of likelihood method. They assumed that the experimental noises are white, Gaussian and two-by-two independent. This method, called also BCGO method, is shown to be the most insensitive to noise in [24].

Alternatively to the redundant measurements techniques, Casem et al. [23] proposed the use of one strain and one velocity measurement at the same cross-section. The two measurements give two independent equations for all frequencies. Hence, the initial noise is less amplified.

To summarize, the two-strain methods are noise sensitive $[12-18,21,22]$. In more general case, unless one strain and one velocity measurement are recorded at the same cross-section, two-measurement (strain or velocity measurements) techniques amplify noise. To reduce noise effects, one can either use one strain measurement and one velocity measurement at the same cross-section [23] or take advantage of redundant measurements [20,24]. In this paper the BCGO method is used for the measurement of the load applied by the servo-hydraulic machine because of its multiple assets which comply with situation under study. These assets can be summarised as follows:

(1) It is the most noise-insensitive wave separation technique.

(2) Wave dispersion effects are taken into account.

(3) It does not give rise to any restraint on the test duration (the test duration is increased to some seconds [26] which is some thousand times more than the duration of a conventional SHB). 
(4) It can be used with strain and (or) velocity measurements.

(5) The error on measurements can be taken into account individually, i.e., if the measurements have different noise levels, a weight inversely proportional to the noise level can be attributed to each measurement. Therefore, the less-noisy measurements contribute more than the others to the recovered force and velocity [19].

(6) The accuracy of the method can be highly improved by increasing the number of the redundant measurements.

The BCGO method will be detailed in the subsection 3.3.

\section{Testing with modified servo-hydraulic machine}

\subsection{Experimental set-up}

An MTS 819 servo-hydraulic machine, with maximum force capacity of $25 \mathrm{kN}$ and maximum velocity of $16 \mathrm{~m} / \mathrm{s}$, is used. In the standard configuration, a piezoelectric force sensor is fixed on the upper cross head of the machine (Fig. 1a). For low strain rate tests $(\dot{\epsilon} \prec 10 /$ s), this sensor gives reliable measurements. However, for higher strain rates the force measurement is degraded by oscillations which are induced by the elastic response of the machine [2]. To overcome this problem, an alternative force measurement is proposed (Fig. 1b). A steel bar, $0.82 \mathrm{~m}$ in length and $16 \mathrm{~mm}$ in diameter, is attached to the upper cross head of the machine. Three gauge stations are cemented on the bar at $x_{1}=0.2 \mathrm{~m}, x_{2}=0.38 \mathrm{~m}$ and $x_{3}=0.64 \mathrm{~m}$, where the $x$-origin is taken at the cross-head. The first and the third gauges are $200 \mathrm{~mm}$ and $180 \mathrm{~mm}$ away from the bar ends, respectively. These gauge positions en- 
sure that the measured strains are in accord with the one-dimensional wave propagation theory. A simplified schematic of the new set-up is given in Fig. 2.

\subsection{Experimental procedure}

Firstly, the strain gauges are calibrated on the SHB set-up. The three-gaugeMTS bar is shot by a $200 \mathrm{~mm}$ long striker at an impact velocity of approximately $10 \mathrm{~m} / \mathrm{s}$. The nominal force should be $N_{\text {nom }}=E_{b} A_{b} V_{s} / 2 c_{0}$ where $E_{b}, A_{b}$, $V_{s}$ and $c_{0}$ are the bar Young's modulus, the bar cross-sectional area, the impact striker velocity and the sound velocity of the bar, respectively. Let $U_{i}$ be the nominal voltage measured on the $\mathrm{i}^{\text {th }}$ gauge, the calibration coefficient for the same gauge is $\alpha_{i}=E_{b} A_{b} V_{s} / 2 c_{0} U_{i}$. This calibration test is repeated three times. The mean values are $\alpha_{1}=41580 \mathrm{~N} / \mathrm{V}, \alpha_{2}=41746 \mathrm{~N} / \mathrm{V}$ and $\alpha_{3}=41018$ $\mathrm{N} / \mathrm{V}$. In the case of our study the calibration procedure was done just once.

After the calibration step, the bar is mounted on the upper cross head. At the same time, a speckle pattern is painted on each specimen. After 20 minutes the specimen can be tested.

The specimen to be tested is attached to the bar and to the lower cross head via two identical attaching devices(Fig. 1b). One attaching device is screwed on the lower end of the bar and the other is screwed on the lower cross head. The specimen is fixed to each device by two cap screws. A couple of approximately 30 N.m is applied to each screw.

All measurements, gauges and video camera, are synchronised. The trigger is given by the start-up of the lower cross head. The flecked sample is illumi- 

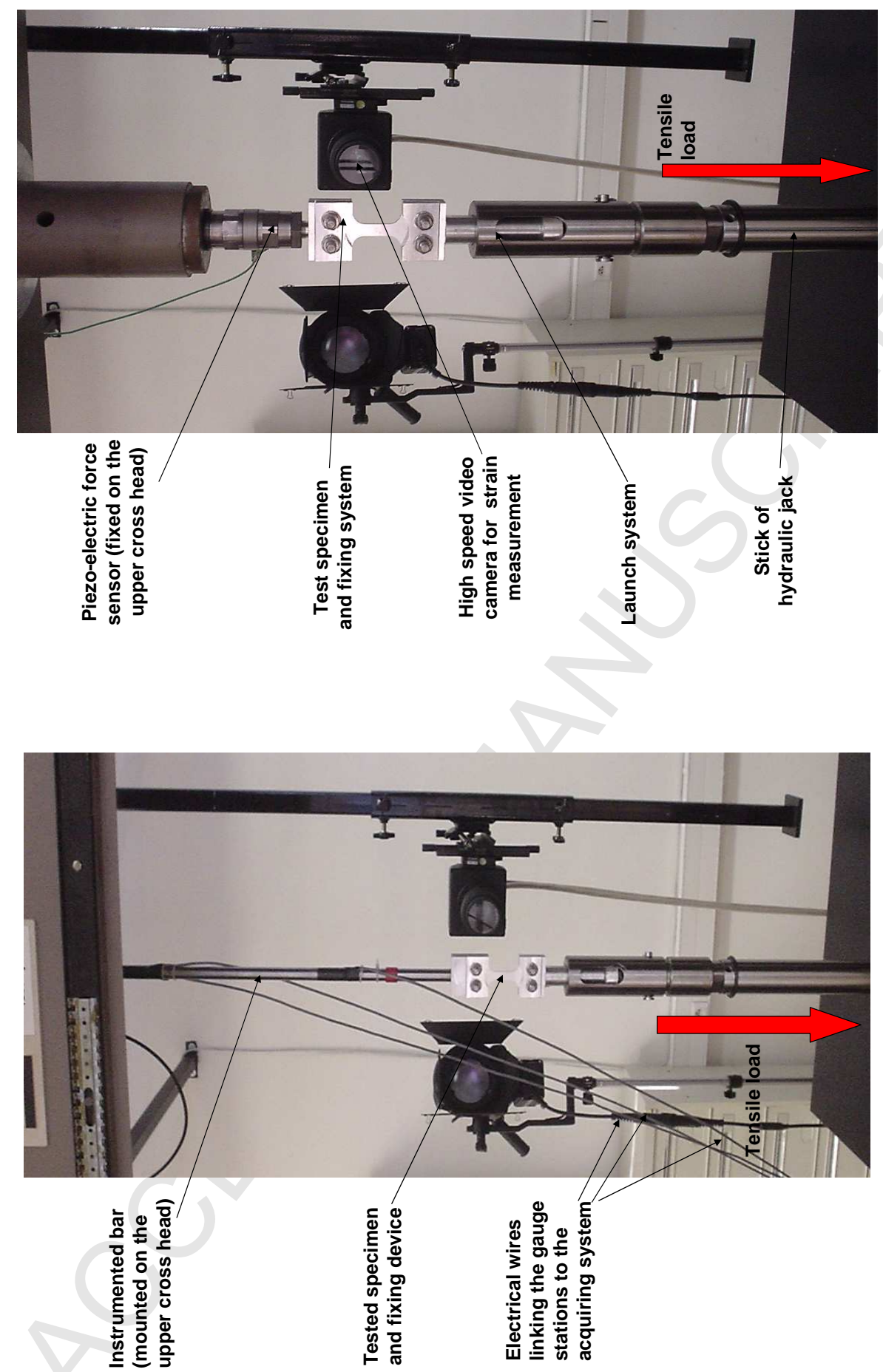

Fig. 1. Experimental set-up: (a) original set-up with piezoelectric force transducer, and (b) modified set-up with instrumented bar 
Fig. 2. A simplified schematic of the modified servo-hydraulic machine nated with a coherent light. Photographs of the flecked sample are taken with a frequency of 24000 images/s and a resolution of $512 \times 128 \mathrm{pix}^{2}$.

\subsection{Analysis}

The assessment of the tested material behaviour is equivalent to measuring the true strain-true stress relationship. The true strain is computed via a high speed video camera (PHOTRON Ultima APX) and a DSP (Digital Speckle 
Photographs) technique. Photographs are taken each $41.67 \mu s$. A small region (the useful region) in the middle of the specimen photography taken at a time $t$ is compared to the same region of a reference photography (taken at $t=0)$. Then a digital cross-correlation algorithm is applied to the two selected regions $[10]$ and calculates the in-plane displacement field. The nominal and true (logarithmic) longitudinal strain are then deduced from the displacement field using the conventional relation of continuum mechanics. In our study, the recorded photographs are analysed by the ICASOFT software developed at INSA Lyon [31].

The true stress in the sample is deduced from the force measurement assuming volume conservation. The BCGO method is applied to recover the force at the end of the bar (where the specimen is attached) from the three strain measurements. Below, we will give a brief introduction to this method. For a full development of this technique, the reader is referred to $[19,20,26]$. First, we assume that $N$ strain measurements $\left(\hat{\epsilon}_{j}(t), j=1, . ., N\right)$ are recorded at $x_{j}, j=1, . ., N(N>2)$. These measurements are subject to noise. Noises are assumed to be statistically independent Gaussian white noises with the same variance. This hypothesis was checked by Othman [26]. The maximum of likelihood functional is then proportional to the functional $\mathcal{F}$, which is given by:

$$
\mathcal{F}=\int_{-\infty}^{+\infty} \sum_{j=1}^{N}\left(\hat{\epsilon}_{j}(t)-\epsilon\left(x_{j}, t\right)\right)^{2} d t
$$

Using Parceval relation and Eq. (7), one obtains:

$$
\mathcal{F}=\int_{-\infty}^{+\infty} \sum_{j=1}^{N}\left|\hat{\hat{\epsilon}}_{j}(\omega)-F(\omega) e^{-i \xi(\omega) x_{j}}-D(\omega) e^{i \xi(\omega) x_{j}}\right|^{2} d \omega
$$


The maximization of the likelihood function with respect to $F(\omega)$ and $D(\omega)$ is equivalent to the minimization of the functional $\mathcal{F}$ with respect to the same parameters. The analytical solving of the optimization problem yields:

$$
\begin{aligned}
& F(\omega)=\frac{h_{2}(\omega) E_{1}(\omega)-g_{1}(\omega) E_{2}(\omega)}{h_{1}(\omega) h_{2}(\omega)-g_{1}(\omega) g_{2}(\omega)}, \\
& D(\omega)=\frac{h_{1}(\omega) E_{2}(\omega)-g_{2}(\omega) E_{1}(\omega)}{h_{1}(\omega) h_{2}(\omega)-g_{1}(\omega) g_{2}(\omega)}
\end{aligned}
$$

where $h_{1}(\omega)=\sum_{j=1}^{N} e^{-i(\xi(\omega)-\bar{\xi}(\omega)) x_{j}}, h_{2}(\omega)=\sum_{j=1}^{N} e^{i(\xi(\omega)-\bar{\xi}(\omega)) x_{j}}, g_{1}(\omega)=$ $\sum_{j=1}^{N} e^{i(\xi(\omega)+\bar{\xi}(\omega)) x_{j}}, g_{2}(\omega)=\sum_{j=1}^{N} e^{-i(\xi(\omega)+\bar{\xi}(\omega)) x_{j}}, E_{1}(\omega)=\sum_{j=1}^{N} \tilde{\hat{\epsilon}}_{j}(\omega) e^{i \bar{\xi}(\omega) x_{j}}$ and $E_{2}(\omega)=\sum_{j=1}^{N} \tilde{\hat{\epsilon}}_{j}(\omega) e^{-i \bar{\xi}(\omega) x_{j}}$. Eqs. (9-10) give the forward and the downward waves as functions of the redundant strain measurements and the wave dispersion relation. This last parameter only depends on the bar geometry and material. It is separately determined using the technique presented in [32]. Hence, the force at the lower bar end is deduced using Eqs. (3,9-10).

The BCGO method is based on a uniform bar model. However, the attaching device makes an impedance variation on the wave propagation way between the bar and the sample. Hence oscillations may error the force measurement. Fortunately, the wave energy appears at rather low frequencies. Furthermore, the maximum in-plane dimension of the attaching device is of some millimeters. Consequently, the lowest wavelength is much greater than the maximum dimension of the attaching device. We can conclude that the elastic response of the material can be neglected. Only, its inertia will be considered.

Let $N_{B C G O}$ be the force calculated by the BCGO method at the lower bar end and let $V_{B C G O}$ be the velocity calculated by the same method and at the same end using Eqs. (2,9-10). Then, the actual 
force applied to the specimen is:

$$
N_{\text {sample }}=N_{B C G O}-m_{a d} \frac{\partial V_{B C G O}}{\partial t}
$$

where $m_{a d}$ is the mass of the attaching device.

4 Results and discussion

\subsection{Results}

Experiments are carried out on samples cut from a commercial Al 2017 T4 sheet which is $2.5 \mathrm{~mm}$ thick (see Fig. 3). The parameters of these tests are detailed in Tab. 1. An example of the recorded strains at the gauge stations is given in Fig. 4. Strain measurements show clearly important oscillations due to the elastic response and the inertia of the measurement system (the bar + the specimen attaching device).

Let us now consider the impact of using the BCGO method on force recovering. In Fig. 5, we compare the recovered force, at the attaching device-bar interface, with the force directly measured by the gauge at the station 1 . The initial oscillations on the measured force are highly reduced. Nevertheless, they are not completely removed. Indeed, the BCGO method, as all wave separation techniques cited above, only takes into account the elastic response and the inertia of the bar and not those of the attaching device. To overcome this problem, the actual force applied to the specimen is corrected using Eq. (11) which includes attaching device inertia. The resulting force has much 


\begin{tabular}{|c|c|c|c|c|}
\hline Test & Cross-beam & Strain rate & & Force \\
label & velocity $(\mathrm{m} / \mathrm{s})$ & $\left(\mathrm{s}^{-1}\right)$ & Geometry & measurement \\
\hline B1 & 0.1194 & 5.97 & Fig. 3 & Bar \\
\hline B2 & 0.1206 & 6.03 & Fig. 3 & Bar \\
\hline B3 & 2.36 & 118 & Fig. 3 & Bar \\
\hline B4 & 2.44 & 122 & Fig. 3 & Bar \\
\hline P1 & 2.32 & 116 & Fig. 3 & Piezo-electric \\
\hline P2 & 2.18 & 109 & Fig. 3 & Piezo-electric \\
\hline QS & $510^{-5}$ & 0.001 & NF EN ISO 527 & Quasi-static machine \\
\hline
\end{tabular}

Table 1

Parameters of the experimental tests

lower oscillating behaviour (see Fig. 6). The remaining oscillations are due to the neglected elastic response of the attaching device. They can be removed by considering the impedance mismatch between the bar and the attaching device [33]. However, this treatment is out of the scope of this paper since these oscillations have low magnitude and can be neglected.

In Fig. 7, the stress-strain curves obtained by the old and the new techniques are compared. The new technique shows less-oscillating behaviour at intermediate strain rates (about 100/s). In Fig. 8, the stress-strain curves obtained by the new technique at a strain rate of $6 / \mathrm{s}$ are compared to a stress-strain curve obtained by a conventional quasi-static machine at a strain rate of $0.001 / \mathrm{s}$. A very good agreement is found between the two techniques since aluminium is insensitive to the deformation rate in this range of strain rates. 


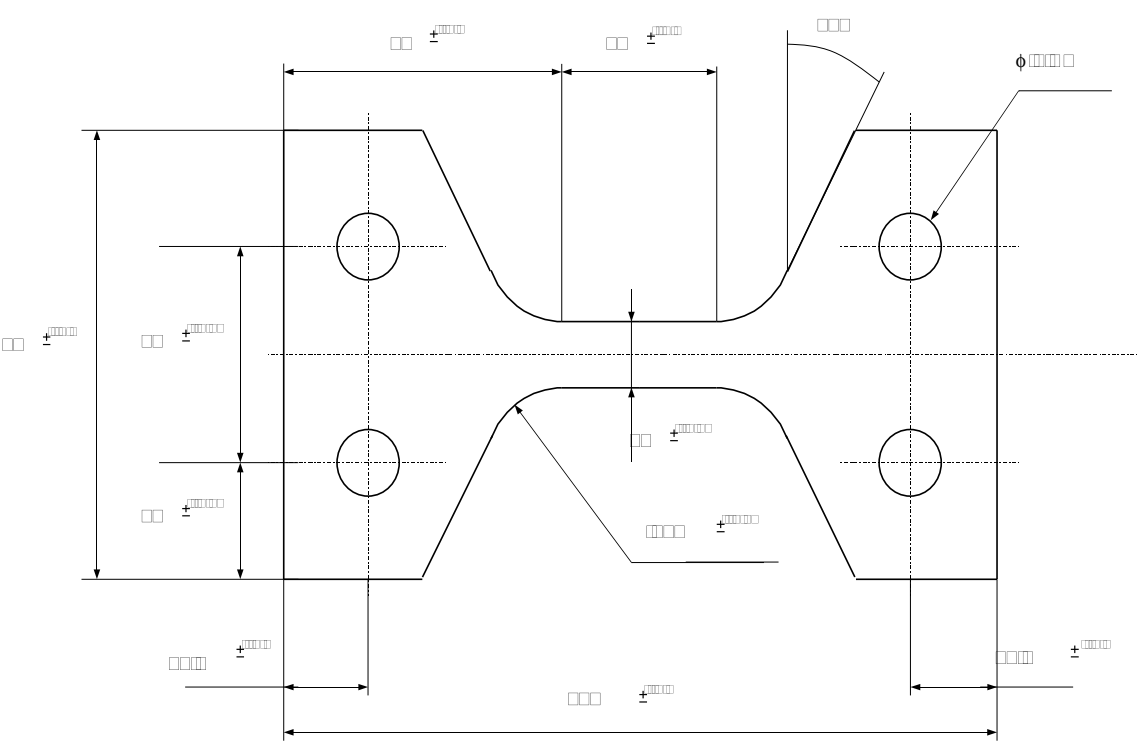

Fig. 3. Geometry of the sample

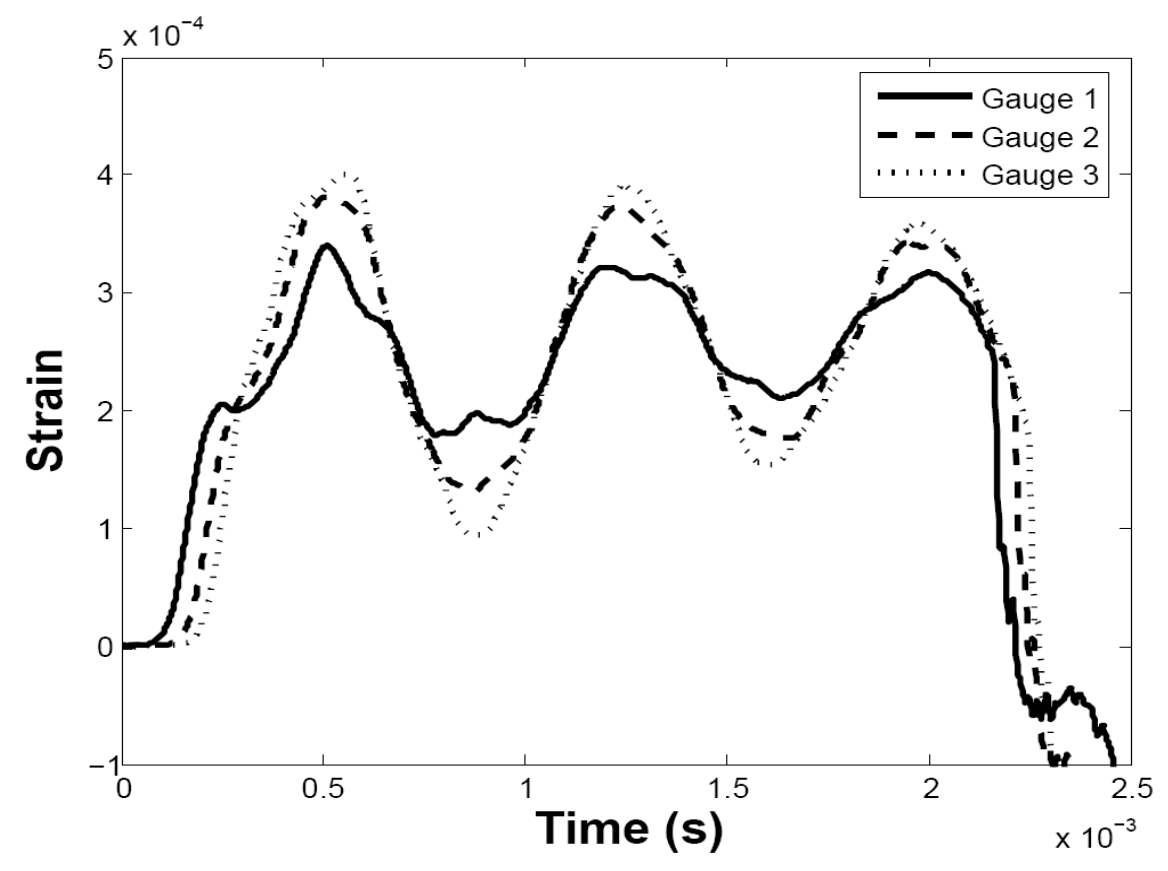

Fig. 4. Measured strains on the gauge stations (test B4) 


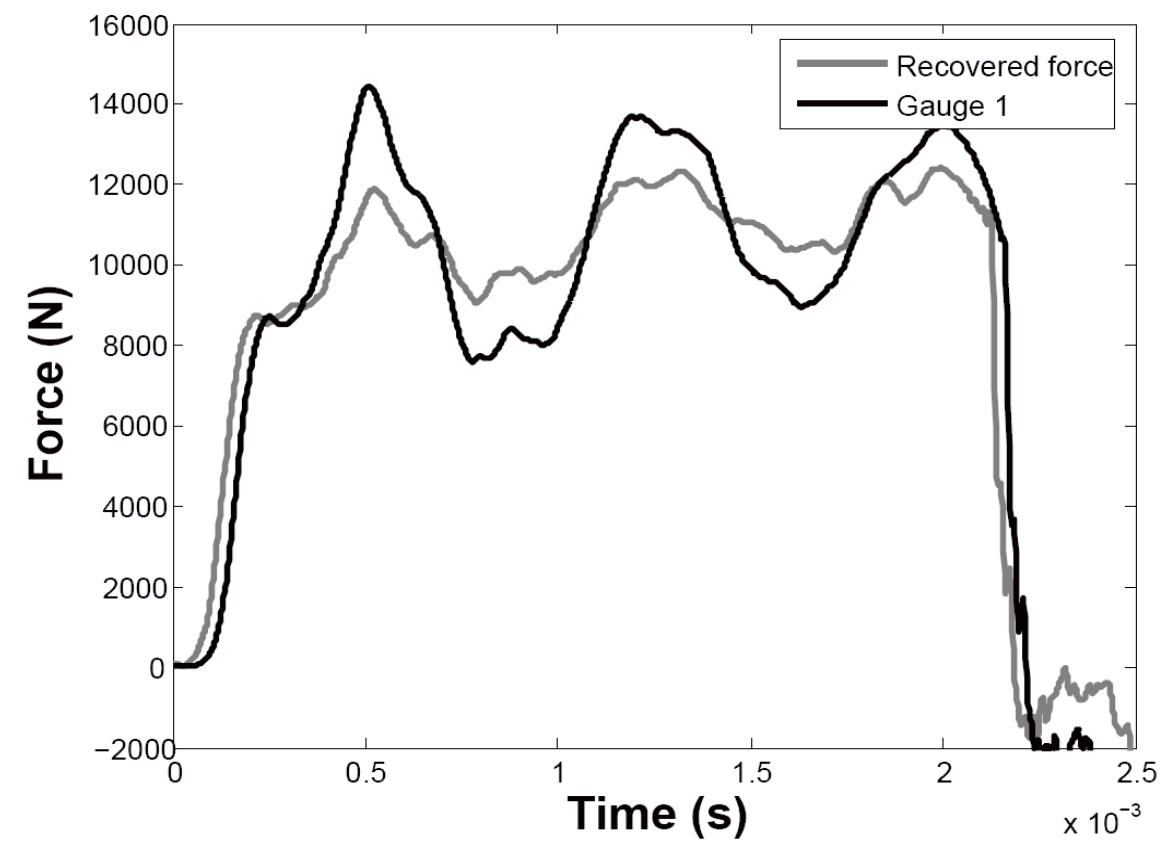

Fig. 5. The force at the attaching device-bar interface compared to the measured force at gauge 1

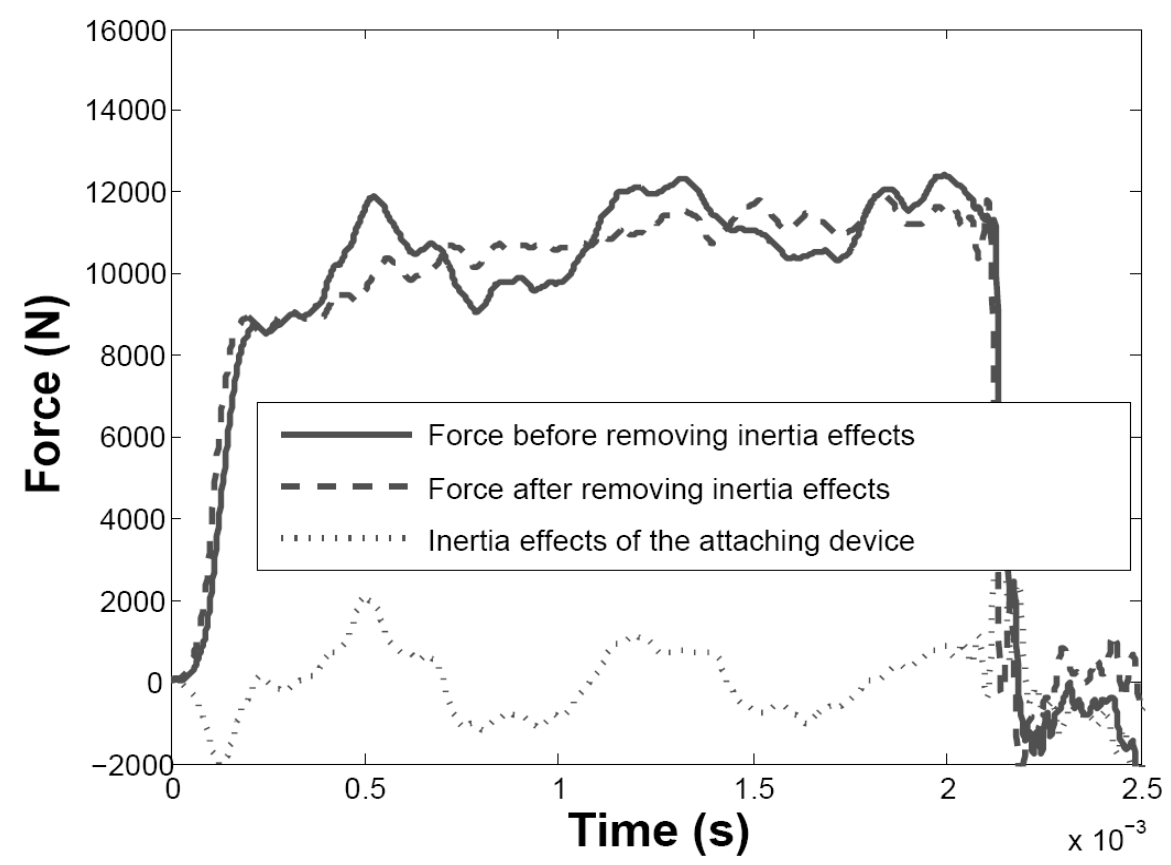

Fig. 6. The measured force before and after removing the inertia effects of the attaching device 


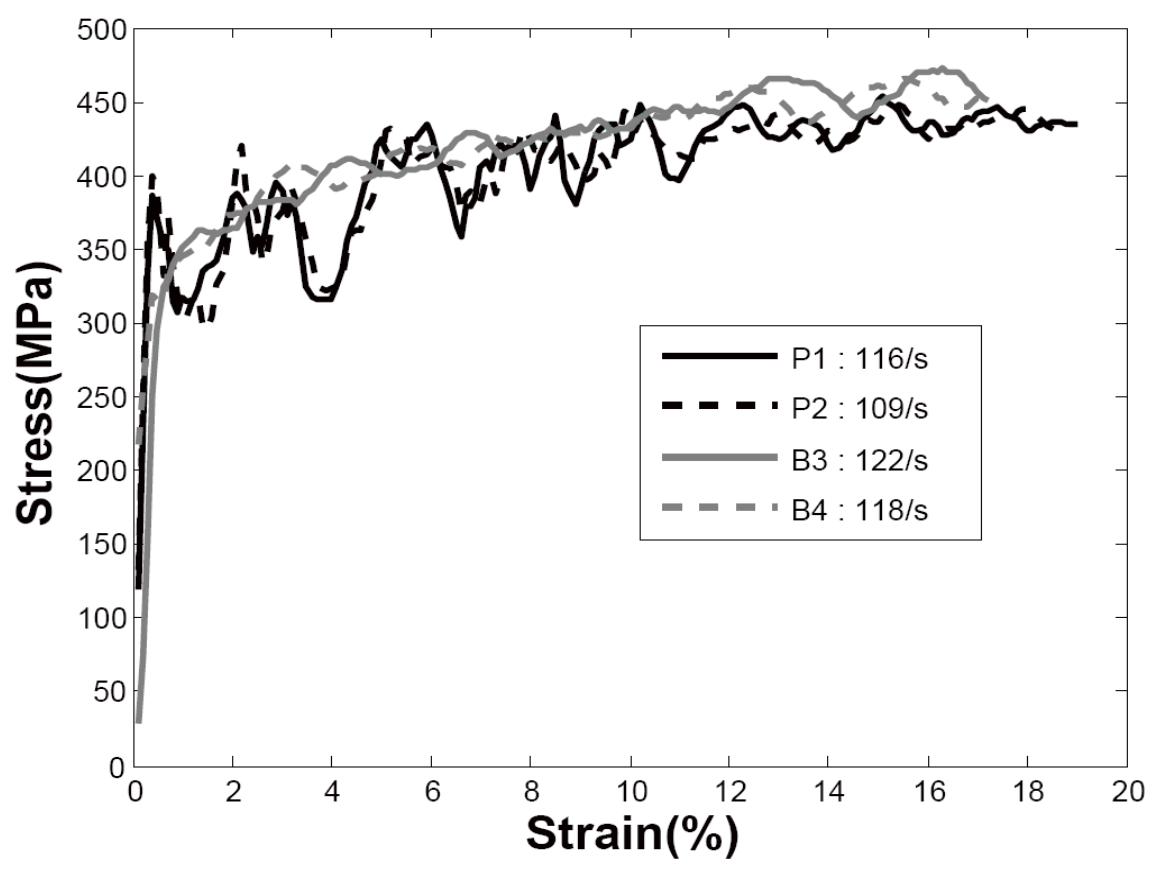

Fig. 7. Stress-strain relation for aluminium obtained by the piezoelectric force transducer method (P1 and P2) and by the bar method (B3 and B4)

It is worth noticing that the test duration is considerably increased with the new technique. Indeed, the used bar is $820 \mathrm{~mm}$ long and has a wave velocity of approximately $5100 \mathrm{~m} / \mathrm{s}$. Using the same bar, the test duration will be $160 \mu \mathrm{s}$ in the case of a conventional Hopkinson bar. It will be less than $320 \mu \mathrm{s}$ in the case of the hybrid technique [3]. At a strain rate of approximately 100/s, the maximum strain measured on the specimen will be less than $1.6 \%$ in case of the first technique and less than $3.2 \%$ in the case of the second technique. Using the new bar-like method, the test duration is theoretically unlimited. It is $1.5 m s$ in the tests B3 and B4 and $37 m s$ in the tests B1 and B2. Besides, the maximum measured strain is about $20 \%$. 




Fig. 8. Stress-strain relation for aluminium obtained by a conventional quasi-static machine (QS) and by the bar method (B1 and B2)

\subsection{Discussion}

In this paper, an indirect and long duration force measurement, developed in the past with a Split Hopkinson bar technique [20], is now applied to servohydraulic machines. Many points can be discussed about this new application:

(1) The rigid mass model is valid if the test duration, $\Delta t$, is much higher than the time needed by the wave to go through the attaching device. Let $\epsilon_{\max }$ and $\dot{\epsilon}$ be the maximum strain induced in the tested specimen and the average strain-rate during the test, respectively. Then the validity of the rigid mass model can be written as follows:

$$
\Delta t \approx \frac{\epsilon_{\max }}{\dot{\epsilon}}>>\frac{d_{\max }^{(a d)}}{c_{\min }^{(a d)}} \approx \frac{d_{\max }^{(a d)}}{c_{0}^{(a d)} / 2}
$$


where $d_{\max }^{(a d)}, c_{\min }^{(a d)}, c_{0}^{(a d)}$ the maximum dimension, the minmum wave speed and the sound speed, respectively, of the attaching device. By introducing a factor 100 and replacing $>>$ by $>$, Eq. (12) yields:

$$
\dot{\epsilon} \leq \frac{c_{0}^{(a d)} \epsilon_{\max }}{200 d_{\max }^{(a d)}} .
$$

(2) The second point to be adressed is the difference of complexity between the wave separation method and the rigid body model.

The wave separation methods take into account the stiffness and the inertia of a uniform bar. The attaching device has non uniform crosssection. Consequently, wave propagation is affected by the impedance variation. Furthermore, the uniform bar wave separation methods can not be applied to the whole system (the bar + the attaching device). In this work the force applied to the specimen is computed in two steps. In the first step, we calculate the force at the bar-attaching device interface using the BCGO method. Secondly, we deduce the force applied to the sample assuming a rigid-mass model for the attaching device. The first model is complex but necessary, whereas the second one is rather simple but sufficient.

The force measurement is based here on strain measurement and elasticity. A long bar is used to avoid 3D effects because only the axial force is needed here. Furthermore, transient waves become significant and should be taken into account at intermediate strain rates. This point leads also to long bars because transient waves in long bars are the best understood and because their analysis is the simplest. Therefore, inertia and stiffness of the measuring device can be corrected. Finally, the better way to measure force at intermediate strain rates (and higher), using 
strain measurements, is that using long (visco)-elastic bars. In addition, the incident and reflected waves contribution are measured together with the strain measurements, mainly at intermediate strain-rate tests. Hence, the contributions of the two waves must be separated to calculate the force at the bar end. Therefore, wave separation techniques, despite their complexity, should be used.

The rigid model of the grip is quite simple. However, it is valid for test durations that are much greater than the time needed by the wave to go through the attaching device. We show in Eq. (13), that this model is valid at least for strain-rates $\dot{\epsilon} \leq$ $C_{0, a d} \epsilon_{\max } / 200 d_{\max }$. Taking $C_{0, a d}=5000 \mathrm{~m} / \mathrm{s}, \epsilon_{\max }=0.2$ and $d_{\max }=$ $22 \mathrm{~mm}$, this model is valid in the range of strain-rates of some hundreds per second.

Even though the methods used in the two steps are of different complexity, the corresponding oscillation elimination is of the same order. In fact, wave separation methods eliminate approximately $50 \%$ of the oscillations and the rigid body model the same amount (see Fig. 9). This shows that the rigid mass model for the attaching device is efficient.

(3) In this work, the force applied to the specimen is computed in two steps. Alternatively, it can be calculated within one step by taking into account both inertia and stiffness of the bar and the attaching device. Therefore, the bar and the attaching device can be modelled as a whole non-uniform bar as suggested in [33]. Nevertheless, the rigid body model gives good results in the range of strain-rates of some hundreds per second. Indeed, the remaining oscillations have low level.

(4) The BCGO wave separation method is an indirect force measurement. Therefore, it can be applied to any loading system. It was first applied 


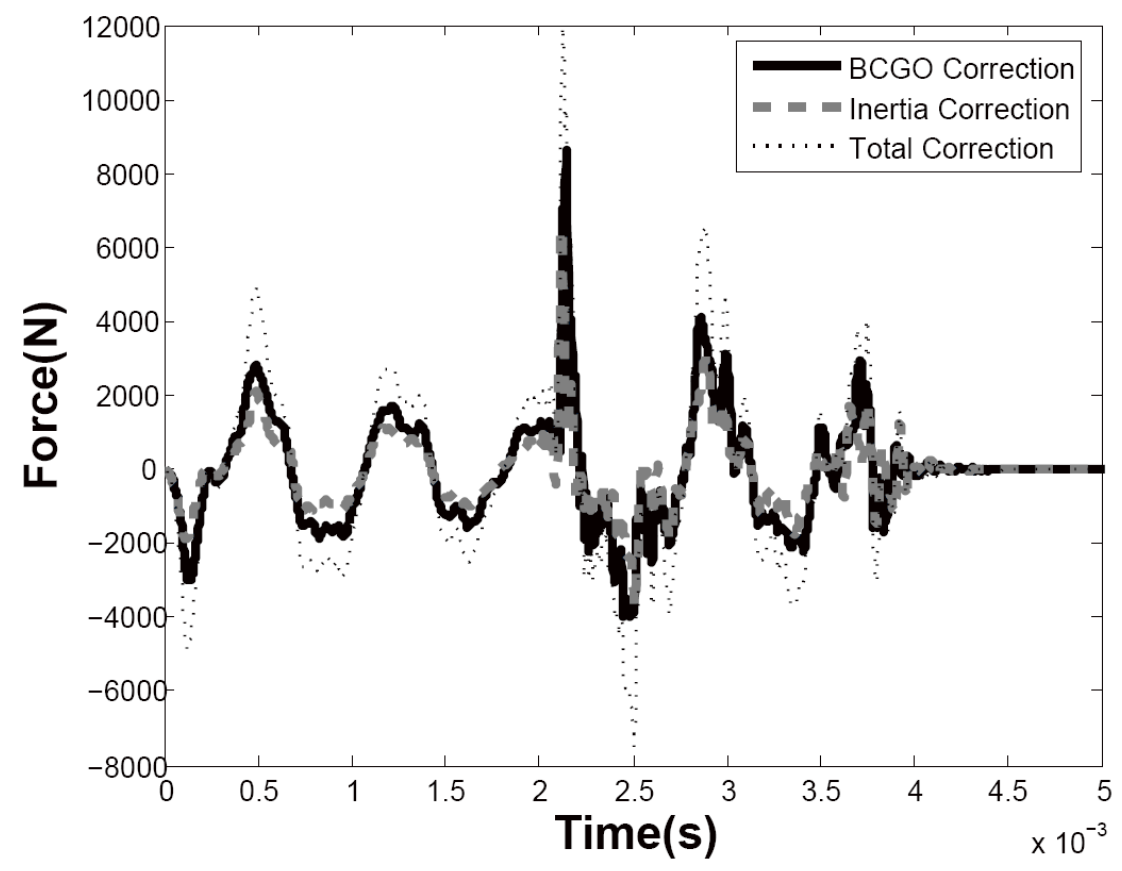

Fig. 9. BCGO and rigid mass corrections (Test B3)

to Split Hopkinson bar technique. In this work, we successfully applied it to servo-hydraulic machines. It can also be applied, for example, to dropweight towers.

(5) In this work, the measured strains are analysed by the BCGO wave separation method. However, one can carry out strain-measurement analysis with any wave separation technique. Nevertheless, the section 2 clearly shows that the BCGO method is the least noise sensitive.

(6) The test duration is theoretically unlimited. Actually, it depends on the acquiring system which is limited by the maximum storage capacity. This would be a problem to the quasi-static tests. Nevertheless, only low-frequencies are excited when velocity tends to quasi-static values. Therefore, low sampling rates are required and with the same storage capacity, it is possible to have long acquiring duration. Consequently, the bar method developed in this work can also be applied to quasi-static 
tests.

(7) The method developed in this paper is applied to a tensile test. It can be easily applied to a compressive test. However, a special care should be taken to prevent buckling.

\section{Conclusion}

Force measurement is one of the major concerns in impact engineering. In this paper, we presented a bar-like force measurement for servo-hydraulic machines. In the first part, wave separation techniques were reviewed. Then we showed that the BCGO method [20] is the most insensitive to the experimental noise. In the second part, this method is applied to improve the hybrid technique presented in [3]. Indeed, intermediate strain rate tests are carried out without any limitation on the test duration. The oscillations, due to the elastic behaviour and the inertia of the measuring set-up, are almost totally removed. Results obtained at a strain rate of approximately $120 / \mathrm{s}$ are more accurate than those obtained by a piezoelectric cell at the same range of strain rates. Moreover, the results obtained at a strain rate of approximately $6 / \mathrm{s}$ are in line with those obtained by a conventional quasi-static machine.

\section{References}

[1] Gary G. Comportement dynamique des métaux à grande vitesse de déformation. Modélisation. Techniques de l'Ingénieur 2001;BM 7 176:1-10.

[2] Diot S, Guines D, Gavrus A, Ragneau E. Two-step procedure for identification 
of metal behavior from dynamic compression tests. Int $\mathrm{J}$ Impact Eng 2007;34:1163-1184.

[3] LeBlanc MM, Lassila DH. A hybrid technique for compression testing at intermediate strain rates. Exp Tech 1996;20:21-24.

[4] Lengyel B, Mohitpour M. Dynamic stress/strain data to large strains. J Inst Metals 1972;100:1-5.

[5] Holzer AJ. A technique for obtaining compressive strength at high strain rates using short loading cells. Int J Mech Sci 1978;20:553-560.

[6] Field JE, Swallowe GM, Pope PH, Palmer. Mechanical properties at high rates of strain. Institute of Physics conference Series 70 (ICP, London 1984), pp 381384.

[7] Groves SE, Sanchez RJ, Lyon RE, Brown AE. High strain rate effects for composite materials. Composites Materials Testing and Design, ed. E.T. Camponeschi 1993;11 ASTM STP 1206.

[8] Hamdan S, Swallowe GM. The strain-rate and temperature dependence of the mechanical properties of polyetherketone and polyetheretherketone. J Mater Sci 1996;31:1415-1423.

[9] Roland CM, Twigg JN, Vu Y, Mott PH. High strain rate mechanical behavior of polurea. Polymer 2007;48:474-478.

[10] Field JE, Walley SM, Proud WG, Goldrein HT, Siviour CR. Review of experimental techniques for high rate deformation and shock studies. Int $\mathrm{J}$ Impact Eng 2004;30:725-775.

[11] Guégan, LeBreton D, Pasco F, Othman R, Le Corre S, Poitou A. Metallic energy absorbing inserts for formula one tyre barriers. Proc Inst Mech Eng Part D, J Automobile Eng 2008; in press. 
[12] Lundberg B, Henchoz A. Analysis of elastic waves from two-point strain measurement. Exp Mech 1977;17:213-218.

[13] Yanagihara N. New measuring method of impact force. Bull Jpn Soc Mech Engng 1978;21:1085-1088.

[14] Zhao H, Gary G. A new method of wave separation for application to dynamic testing. C. R. Acad. Sci. Paris 1994;319:987-992.

[15] Zhao H, Gary G. A new method for the separation of waves. Application to the SHPB technique for an unlimited measuring duration. J Mech Phys Solids 1997;45:1185-1202.

[16] Bacon C. Separating waves propagating in an elastic or viscoelastic Hopkinson pressure bar with three-dimensional effects. Int J Impact Eng 1999;22:55-69.

[17] Park SW, Zhou M. Separation of elastic waves in Split Hopkinson Bars using one-point strain measurements. Exp Mech 1999;39:287-294.

[18] Jacquelin E, Hamelin P. Block-bar device for energy absorption analysis. Mech Syst Signal Process 2001;15:603-617.

[19] Othman R, Bussac MN, Collet P, Gary G. Séparation et reconstruction des ondes dans les barres élastiques et viscoélastiques à partir de mesures Redondantes. C R Acad Sci Sér IIb 2001;329:369-376.

[20] Bussac MN, Collet P, Gary G, Othman R. An optimisation method for separating and rebuilding one-dimensional dispersive waves from multi-point measurements. Application to elastic or viscoelastic bars. J Mech Phys Solids 2002;50:321-350.

[21] Zhao PJ, Lok TS. A new method for separating longitudinal waves in a large diameter Hopkinson bar. J Sound Vib 2002;257:119-130. 
[22] Meng H, Li QM. An SHPB set-up with reduced time-shift and pressure bar length. Int J Impact Eng 2003;28:677-696.

[23] Casem DT, Fourney W, Chang P. Wave sepration in viscoelastic pressure bar using single point measurements of strain and velocity. Polym Test 2003;22:155164.

[24] Jacquelin E, Hamelin P. Force recovered from three recorded strains. Int J Solids Struct 2003;40:73-88.

[25] Zhao H, Abdennadher S, Othman R. An experimental study of square tube crushing under impact loading using a modified large scale SHPB. Int J Impact Eng 2006;32:1174-1189.

[26] Othman R. Extension du champ dapplication du systme des barres de Hopkinson aux essais à moyennes vitesses de déformation. Ph.D. Thesis, Ecole Polytechnique, France, 2002.

[27] Othman R, Bussac MN, Collet P, Gary G. Testing with SHPB from quasi-static to dynamic strain rates. Journal de Physique IV 2003; 110:397-402.

[28] Othman R, Gary G. Testing aluminum alloy from quasi-static to dynamic strain-rates with a modified Split Hopkinson Bar method. Exp Mech 2007;47:295-299.

[29] Quirion Y, Lesaffre AS. Hydraulic machine tests for compression of a quasibrittle material at medium strain rate. Journal de Physique IV 2006;134:553558.

[30] Yang CY. Solution of an inverse vibration problem using a linear least-squares error method. Appl Math Modelling 1996;20:785-788.

[31] http://icasoft.insa-lyon.fr 


\section{ARTICLE IN PRESS}

[32] Othman R, Blanc RH, Bussac MN, Collet P, Gary G. Identification de la relation de dispersion dans les barres. C R Mécanique 2002;320:849-855.

[33] Othman R. Wave separation in non-uniform Hopkinson bars using redundant measurements. Journal de Physique IV 2006;134:571-576. 


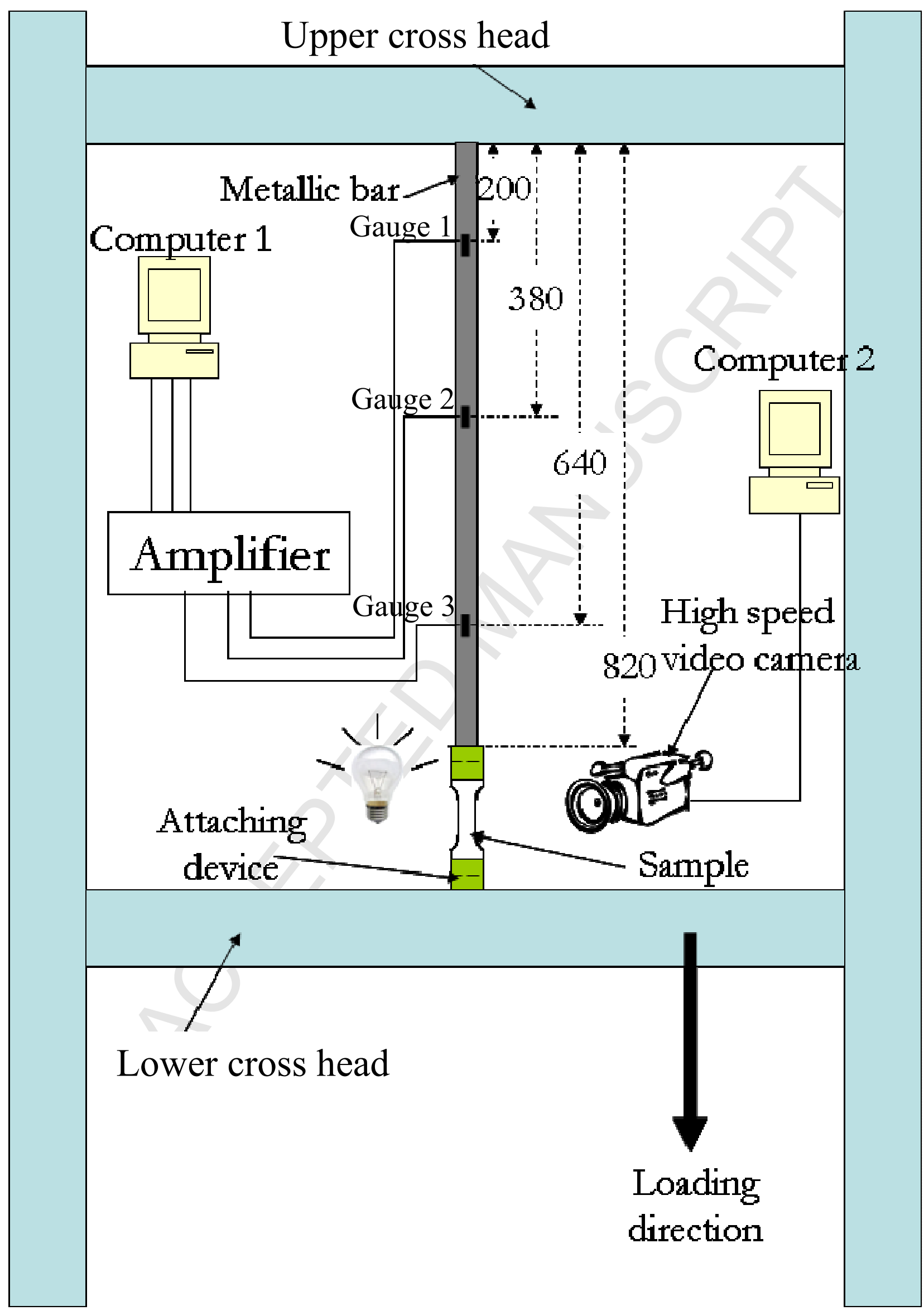

